\title{
RELAÇÃO ENTRE GASTO PÚBLICO EM EDUCAÇÃO E DESEMPENHO EDUCACIONAL: UMA ANÁLISE DOS MUNICÍPIOS DO NORDESTE
}

Georgiana Karla Oliveira de Morais ${ }^{1}$ Maria Elza de Andrade ${ }^{2}$ Rodolfo Ferreira Ribeiro Costa ${ }^{3}$ Francisco Soares de Lima $^{4}$

RESUMO: O presente trabalho faz uma análise da relação entre o gasto público em educação e desempenho educacional, mais propriamente, verifica se um maior gasto em educação nos municípios do Nordeste leva a um maior aprendizado de seus alunos, para o ano de 2015. Para fins de obtenção dos resultados o método aplicado foi o de regressão quantílica. Foram utilizados como medida de desempenho educacional a proficiência em teste de português e matemática, aplicados em alunos matriculados no $5^{\underline{a}}$ ano da rede municipal do Nordeste. Como medida de gasto foi considerada a despesa em educação ponderada por aluno matriculado. Também foram testados os efeitos do PIB per capita municipal, escolaridade das mães, formação acadêmica dos professores e infraestrutura escolar. A relação entre o gasto público em educação e desempenho educacional não apresentou resultados claros, porém a formação acadêmica dos professores apresentou uma forte relação com o desempenho educacional.

Palavras-Chave: Gasto público. Desempenho educacional. Economia da Educação.

\footnotetext{
${ }^{1}$ Graduanda em Economia pela Universidade Estadual do Rio Grande do Norte (UERN). E-mail: nick.tafao@hotmail.com

${ }^{2}$ Doutorado em Economia pela Universidade Federal de Uberlândia (UFU) e Professora da Universidade do Estado do Rio Grande do Norte (UERN). E-mail: mariaelza.andrade@gmail.com

${ }^{3}$ Doutor em Economia pela Universidade Federal do Ceará (CAEN/UFC) e Pós-Doutor pela Universidade Federal do Rio Grande do Norte (UFRN), Professor da Universidade do Estado do Rio Grande do Norte (UERN) e do Programa de Pós-Graduação em Economia (PPE/UERN). E-mail: rodolfofrc@yahoo.com.br ${ }^{4}$ Doutor em Economia pela Universidade Federal do Ceará (CAEN/UFC), Professor da Universidade do Estado do Rio Grande do Norte (UERN) e do Programa de Pós-Graduação em Economia (PPE/UERN). E-mail: fsoaresdelima@yahoo.com.br
} 


\section{INTRODUÇÃO}

A Constituição Federal de 1988 (CF/1998) e a Lei de Diretrizes e Bases da Educação Nacional (LDB/96) estabelecem que o sistema educacional brasileiro tenha como característica organizacional a divisão de competências e responsabilidades entre as esferas do governo. Tal divisão também se aplica ao financiamento e à manutenção dos diferentes níveis, etapas e modalidades da educação e do ensino.

Segundo a Constituição Federal de 1988 (CF/1998), a receita dos impostos oriundos da união, dos estados, do distrito federal e dos municípios; de transferências constitucionais; da contribuição social do salário-educação, entre outros, compõem o fomento da educação pública brasileira.

A primeira década pós constituição de 1988 caracterizou-se por uma trajetória crescente dos gastos em educação. Porém, é importante ter conhecimento da eficiência econômica na alocação desses recursos, pois não necessariamente um aumento do gasto público em educação, irá implicar emç. um aumento da qualidade da educação e melhores níveis de desenvolvimento econômico e social.

Diante disso, os gastos eficientes em educação permitem observar a qualidade entre a educação, recursos públicos e indicadores sociais, principalmente para a formulação de políticas públicas. Ferramenta indispensável para que os atores educacionais possam compreender a situação educacional.

De acordo com Almeida (2001), a formulação de políticas educacionais depende do conhecimento do tamanho do gasto realizado, medido em porcentual do PIB, das prioridades estabelecidas a serem atendidas, medidas por meio do gasto por aluno, pois é esse valor que mostra o investimento unitário realizado pelo governo em cada nível de ensino.

A política educacional de um país não está condicionada apenas ao governo central. Existem fatores externos, entre eles, a autodeterminação dos entes federados (estados e municípios), as demandas forjadas no campo da economia e do mercado de trabalho, e as que provêm da mobilização de setores reivindicativos da sociedade (FONSECA, 2009). 
Ao longo das últimas décadas houve grande interferência na educação por meio dos planos educacionais do governo, que eram influenciados por múltiplos fatores, dentre eles o próprio encaminhamento do desenvolvimento econômico do país.

Com efeito, a ação educativa deu ênfase a programas e projetos orientados pela esfera econômica, dirigindo a ação escolar para as atividades instrumentais do fazer pedagógico e para a administração de meios ou insumos. A qualidade educacional, por sua vez, foi sendo legitimada pela competitividade, cuja medida é dada pela boa colocação no ranking das avaliações externas.

O custeio da educação é um tema de extrema importância nas discussões sobre o encaminhamento que se pretende dar à educação em nosso país, posto que, sem os recursos adequados, torna-se inviável atender ao princípio constitucional que assegura uma escola de qualidade para todos os brasileiros.

Problemas como inadequações institucionais, ineficiência administrativa, a falta de um Plano Municipal de Educação com metas claras para serem alcançadas a médio e longo prazo, inviabiliza o pleno funcionamento da educação, e todas essas deficiências são refletidas na sociedade, que sofrem com precariedade estrutural das escolas e transportes, desqualificação técnica dos servidores, insuficiência de merenda e material escolar, entre vários outros problemas.

Diante do exposto, o objetivo do presente trabalho é analisar se um maior gasto em educação nos municípios do Nordeste leva a um maior aprendizado dos alunos. E para fins de desenvolvimento da pesquisa serão expostos o enquadramento geral dos recursos destinados à educação nos municípios do Nordeste; a realização do levantamento dos determinantes do desempenho educacional e, por fim, a verificação se os gastos contribuem de forma positiva para os indicadores educacionais.

Em destaque, realizar-se-á a relação entre gastos educacionais e o desempenho através do modelo de regressões quantílicas, que além de ser robusto a problemas de heterocedasticidade, também é capaz de detalhar os diferentes pontos da distribuição, caracterizando, portanto, de forma mais fiel as heterogeneidades vngentes.

Além desta seção introdutória, este trabalho conta com mais 4 seções. A seguinte discorre sobre uma visão geral dos gastos municipais e os determinantes do 
desempenho educacional; a terceira apresenta a metodologia de regressões quantílica; a quarta trata os resultados; e, por fim, a quinta apresenta as considerações finais.

\section{REFERENCIAL}

\subsection{VISÃO GERAL DOS GASTOS NOS MUNICÍPIOS}

Os municípios vêm ao longo do tempo acumulando mais responsabilidades a respeito da educação no Brasil e por meio das políticas públicas podemos perceber esse fato. A união, os estados, o Distrito Federal e os municípios organizam, em regime de colaboração, o sistema de ensino. Os municípios são responsáveis prioritariamente pelo ensino fundamental e educação infantil.

Um grande passo foi dado em relação à educação com a Lei de Diretrizes e Bases da Educação Nacional - LDB sancionada em 1996. Esta promoveu maior descentralização de decisões e uma mudança de foco, voltando-se mais para a educação básica.

A LDB aumentou para todas as esferas do governo a parcela da arrecadação de impostos vinculados à educação, os municípios aumentaram sua participação para $25 \%$. Vários programas de transferência de renda para famílias de baixa renda, exigindo como contrapartida a frequência escolar das crianças, têm sido realizados ao longo dos últimos anos. (ARVATE, BIDERMAN, 2009).

A estrutura de financiamento distribui-se pelas esferas de governo. A União divide a aplicação de seus recursos na manutenção do sistema federal, na execução de programas próprios e em transferências para os sistemas estaduais e municipais. Os estados, na composição de seus recursos, somam aqueles recebidos da União aos provenientes de suas fontes. E os municípios recebem recursos da União e dos estados, os quais se somam aos seus próprios.

A implementação por lei em 1998 do Fundo de Manutenção e Desenvolvimento do Ensino Fundamental e da Valorização do Magistério (Fundef), segundo a análise histórica de Almeida (2003), elevou os gastos em educação principalmente na esfera municipal. Tendo como inovação o estabelecimento de um critério institucional de 
partilha proporcional ao número de alunos de cada uma das redes estaduais e municipais.

O fundo objetivava garantir recursos para o ensino fundamental, e uma melhora na redistribuição dos recursos alocados para a educação em cada unidade da Federação (UF) de forma mais equitativa (correspondendo, na prática, a 15\% das receitas líquidas dos Estados e municípios).

\subsection{DETERMINANTES DO DESEMPENHO EDUCACIONAL}

A situação dos gastos públicos locais em educação, segundo Castro e Duarte (2008), assim como Almeida (2001), apresenta uma trajetória crescente. Na literatura exterior existe uma controvérsia sobre a relação entre gasto público em educação e desenvolvimento educacional, existe uma corrente que defende a existência da relação e outra defende a sua inexistência.

Partindo desse ponto emerge a relevância da compreender a importância dos insumos educacionais na qualidade da educação, e em qual situação econômica o município pode ser afetado por alterações na quantidade de recursos destinados à educação.

As respostas para tais problemas podem variar dependendo das características dos alunos; das características de suas famílias como a renda familiar abordada por Barros, Mendonça, Santos e Quintaes (2001), e a escolaridade dos pais Barros e Mendonça (1997b); das características das escolas como suas instalações físicas, Soares e Satyro (2008), e o nível de escolaridade dos professores, Felício e Fernandes (2005); como também a situação socioeconômica local, Heyneman e Loxley (19821983).

A contrapartida lidera uma vertente que relaciona os insumos educacionais e as notas dos estudantes em testes padronizados como grande o suficiente para ser pedagogicamente relevante, esta é conduzida por Hedges, Greenwald e Laine (1994, 1996a, 1996b).

Como medida de qualidade da educação admite-se o desempenho dos alunos em testes padronizados, já que de acordo com Hanushek (2006) as habilidades 
cognitivas dos indivíduos são derivadas da escolaridade, sendo um fator contribuinte para a formação de tais habilidades e desempenho educacional.

No tocante aos fatores familiares, a renda familiar, de acordo com Barros, Mendonça, Santos e Quintaes (2001), quanto maior for a disponibilidade deste fator pelas famílias, maior será o investimento em capital educacional, utilizando recursos próprios para viabilizar este tipo de investimento, atenuando os efeitos de imperfeições do mercado de crédito.

O fator escolaridade dos pais leva em consideração a proposição de que, quanto maior for tal nível de escolaridade, maior será a renda permanente da família, podendo disponibilizar uma maior parcela para investimento em educação para seus filhos. De acordo com Barros e Mendonça (1997 b), outro aspecto abordado, relaciona-se à importância do apoio dos pais; quanto mais elevada a escolaridade dos pais, menores as dificuldades e os custos de aprendizagem dos filhos, pois os pais mais escolarizados entendem as dificuldades e necessidades dos filhos no processo de aprendizagem.

Ainda sobre Barros e Mendonça (1997 b), destaca-se também o fator satisfação por parte dos pais, de que independente do investimento futuro, pais mais escolarizados, apenas almejam ver seus filhos escolarizados. A literatura relata fortemente uma maior relevância de fatores familiares em relação aos demais.

Em relação aos fatores de insumos escolares, o salário dos professores pode relacionar-se com sua formação acadêmica e seu tempo de serviço. Como também a formação acadêmica nada pode influenciar nos aspectos não observáveis, didática, dedicação, paciência, que caracterizam um bom professor (FELÍCIO E FERNANDES, 2005).

O fator instalação física das escolas no que remete a número de computadores, existência de laboratórios de informática e laboratórios de ciências, biblioteca, quadras, etc. O estudo de Soares e Satyro (2008) mostra que no Brasil, em relação ao ambiente físico, há sim efeitos sobre os resultados acadêmicos dos alunos, ao contrário do que ocorre nos países desenvolvidos, conforme revela Hanushek para o caso norteamericano. O mesmo afirma que se os gestores não sabem como lidar com esta 
questão, gastam um enorme tempo que poderia ser utilizado em atividades intrinsecamente pedagógicas.

Soares e Clemente (2013) abordaram em seu estudo a ocorrência de duas hipóteses, sendo elas de existência e não existência da relação entre gasto em educação e desempenho educacional e que as duas estão condicionadas a impactos diferentes de acordo com a metodologia e amostra utilizadas. A amostra de uma região em desenvolvimento terá um maior impacto com os aumentos dos insumos em educação do que em uma região desenvolvida, já que estão condicionados a níveis de renda diferentes.

Esse fato toma uma maior relevância para este estudo, pois a região em questão apresenta uma das situações econômicas mais precárias do país. Surgindo então a necessidade da avaliação econômica local e averiguação de ligação com o fator desempenho educacional, podendo ser mensurado em termos do Produto Interno Bruto (PIB) dos municípios do Nordeste.

\section{METODOLOGIA}

Para embasamento científico, será abordado neste trabalho o método de procedimento estatístico de regressão quantílica, prenunciado por Koenker e Bassett (1978). Este, busca medir o comportamento dos indivíduos não médios, estimando várias retas correspondentes a vários pontos percentuais da distribuição, em vez de verificar apenas o valor médio, como é feito numa regressão linear por Mínimos Quadrados Ordinários-MQO.

De acordo com Koenker e Basset (1978), a estimação pelo valor médio apresenta uma visão incompleta da distribuição. A presença de outliers atrapalha a identificação da verdadeira relação das variáveis de interesse, é preciso avaliar quanto cada ponto influencia no ajuste do modelo. A regressão quantílica utiliza a mediana condicional como medida de tendência central, o que torna a regressão mais robusta em resposta aos outliers.

O MQO está associado a uma distribuição normal dos erros, quando há uma assimetria nesta distribuição ou ela possua caudas mais pesadas que a distribuição 
normal, o desempenho do método de estimação fica comprometido, pois as hipóteses do modelo clássico não são atendidas.

O modelo busca estimar o $\tau$-ésimo quantil condicional sobre as diferentes variáveis explicativas, a interpretação para as soluções dos diferentes quantis encontrados, podem ser vistas como variações na variável dependente causadas por mudanças nos regressores em diferentes pontos da distribuição condicional.

Assumindo que esse quantil pode ser expresso como uma função linear dessas variáveis:

$$
Y_{i}=\beta_{0}(\tau)+\beta_{1}(\tau) X_{1 i}+\cdots+\beta_{k}(\tau) X_{k i}+\varepsilon_{i}, \text { onde } \quad 0<\tau<1
$$

Sendo $Y$ a variável dependente, $X_{1} \ldots X_{k}$ as variáveis explicativas, $\beta$ os parâmetros a serem estimados e $\varepsilon \circ$ termo de perturbação aleatória. Dessa forma 0 quantil $\mathrm{Q}$ de ordem $\tau$ de $\mathrm{Y} \mid \mathrm{X}$ é descrito como:

$$
Q_{\tau}(Y \vee X)=\beta_{0}(\tau)+\beta_{1}(\tau) x_{1}+\cdots+\beta_{k}(\tau) x_{k}
$$

O parâmetro pode ser estimado minimizando a seguinte função objetivo, a qual é uma soma ponderada dos desvios absolutos, provendo uma medida robusta, tal que o vetor de coeficientes estimado não é sensível a observações extremas da variável dependente.

$$
\min \sum_{Y_{i} \geq X \beta} \tau \vee Y-X \beta \vee+\sum_{Y_{i} \leq X \beta}(1-\tau) \vee Y-X \beta \vee
$$

Com base em dados secundários, para análise quantitativa, utiliza-se como amostra os municípios da região Nordeste durante o período de 2015. A relação dos gastos públicos em educação e desempenho educacional será dada pelas seguintes variáveis:

- Variável Dependente: o rendimento, no mesmo ano, em testes de desempenho em Português e Matemática de alunos do $5^{\circ}$ ano das redes municipais, utilizando as notas médias obtidas por cada município nos exames, ponderada pelo número de alunos matriculados.

- Variáveis Explicativas: logaritmo natural dos gastos municipais por aluno em educação ao quadrado, logaritmo natural do Produto Interno Bruto (PIB) per capita dos municípios, logaritmo natural da proporção de mães sem ensino superior por 
município, logaritmo natural da proporção de mães com ensino superior por município, logaritmo natural da proporção de professores sem ensino superior por município, logaritmo natural da proporção de professores com ensino superior por município, instalação física das escolas relacionadas à estrutura física escolar (existência cozinha, banheiro, sala da diretoria, sala dos professores, biblioteca, quadra de esportes, laboratório de informática, laboratório de ciências).

A variável instalação física das escolas dispõe a frequência de escolas que apresentavam o mínimo de instalações relevantes para o desenvolvimento das atividades de ensino e aprendizagem por município. As instalações físicas em questão foram banheiro, cozinha, sala da diretoria, sala do professor, biblioteca, quadra de esportes, laboratório de informática e laboratório de ciências.

Foi construída uma escala da variável em questão a qual se inicia na infescolar1 até a infescolar8, visando identificar a frequência de cada grupo de instalações física criadas. Respectivamente a primeira variável infescolar1 é composta pela frequência das escolas que contém todas as instalações físicas citadas relevantes para o estudo por município. As variáveis subsequentes contêm a frequência das escolas com uma instalação física a menos, ordenadas por grau de importância, que corresponde ao inverso da ordem citada anteriormente na descrição das instalações físicas, até que a última variável infescolar8 contenha apenas uma única instalação física.

Tabela 1 - Lista de Variáveis de Instalação Física das Escolas.

\begin{tabular}{cl}
\hline Variável & \multicolumn{1}{c}{ Instalações por Variável } \\
\hline infescolar1 & $\begin{array}{l}\text { Contém cozinha, banheiro, sala da diretoria, sala dos professores, biblioteca, } \\
\text { quadra de esportes, laboratório de informática, laboratório de ciências. } \\
\text { infescolar2 }\end{array}$ \\
infescolar3 & $\begin{array}{l}\text { Contém cozinha, banheiro, sala da diretoria, sala dos professores, biblioteca, } \\
\text { Contém cozinha, banheiro, sala da diretoria, sala dos professores, biblioteca, } \\
\text { quadra de esportes. }\end{array}$ \\
infescolar4 & Contém cozinha, banheiro, sala da diretoria, sala dos professores, biblioteca. \\
infescolar5 & Contém cozinha, banheiro, sala da diretoria, sala dos professores. \\
infescolar6 & Contém cozinha, banheiro, sala da diretoria. \\
infescolar7 & Contém cozinha, banheiro. \\
infescolar8 & Contém cozinha.
\end{tabular}

Fonte: elaboração própria. 
Georgiana Karla Oliveira de Morais, Maria Elza de Andrade, Rodolfo Ferreira Ribeiro Costa, Francisco Soares de Lima

Inserindo as variáveis de interesse no modelo em questão, a relação será quantificada pela seguinte equação de regressão:

$$
\begin{aligned}
& \text { profmed }_{i}=\beta_{0}(\tau)+\beta_{1}(\tau) \text { lngastoeducaluno } 2_{i}+\beta_{2}(\tau) \text { lnpibpercapita }_{i}+\beta_{3}(\tau) \text { lneducmae }_{i}+ \\
& \beta_{4}(\tau) \text { lneducmae } 1_{i}+\beta_{5}(\tau) \text { lnacadpr } 0_{i}+\beta_{6}(\tau) \text { lnacadpr } 1_{i}+\beta_{7}(\tau) \text { infescolar } 1_{i}+ \\
& \beta_{8}(\tau) \text { infescolar } 2_{i}+\beta_{9}(\tau) \text { infescolar } 3_{i}+\beta_{10}(\tau) \text { infescolar } 4_{i}+\beta_{11}(\tau) \text { infescolar }_{\text {inf }_{i}}+ \\
& \beta_{12}(\tau) \text { infescolar } 6_{i}+\beta_{13}(\tau) \text { infescolar } 7_{i}+\beta_{14}(\tau) \text { infescolar } 8_{i}+\varepsilon_{i}
\end{aligned}
$$

A variável dependente foi nomeada profmedlp e profmedmt para a proficiência média dos alunos do $5^{\circ}$ em teste de desempenho de língua portuguesa e matemática por município respectivamente, assim como nas variáveis explicativas o logaritmo natural do gasto municipal em educação por aluno ao quadrado foi nomeado lngastoeducaluno2, o logaritmo natural do PIB per capita municipal lnpibpercapta, o logaritmo natural da proporção de mães sem ensino superior por município lneducmae0, o logaritmo natural da proporção de mães com ensino superior por município lneducmae1, o logaritmo natural da proporção de professores sem ensino superior por município lnacadpr0, o logaritmo natural da proporção de professores com ensino

\begin{tabular}{|c|c|c|}
\hline Variável & Descrição & Efeito \\
\hline lngastoeducaluno 2 & $\begin{array}{l}\text { Gasto municipal em educação por aluno ao } \\
\text { quadrado }\end{array}$ & Positivo/Indiferente \\
\hline lnpibpercapta & Logaritmo natural do PIB per capita municipal & Positivo \\
\hline lneducmae 0 & $\begin{array}{l}\text { Logaritmo natural da proporção de mães sem } \\
\text { ensino superior por município }\end{array}$ & Positivo/Negativo \\
\hline lneducmae 1 & $\begin{array}{l}\text { Logaritmo natural da proporção de mães com } \\
\text { ensino superior por município }\end{array}$ & Positivo \\
\hline lnacadpro & $\begin{array}{l}\text { Logaritmo natural da proporção de professores } \\
\text { sem ensino superior por município }\end{array}$ & Negativo \\
\hline lnacadpr 1 & $\begin{array}{l}\text { Logaritmo natural da proporção de professores } \\
\text { com ensino superior por município }\end{array}$ & Positivo \\
\hline infescolar & $\begin{array}{l}\text { Frequência de grupo de infraestrutura escolar } \\
\text { por município }\end{array}$ & Positivo/Negativo \\
\hline
\end{tabular}
superior por município lnacadpr1, as instalações físicas das escolas infescolar1, fescolar 2 , infescolar 3 , infescolar 4 , infescolar 5 , infescolar6, infescolar 7 , infescolar 8.

\section{Tabela 2 - Efeito das Variáveis Abordadas sobre o Desempenho Educacional}

Fonte: elaboração própria. 


\subsection{FONTE DE DADOS}

A fonte de dados secundários utilizada como medida de qualidade de ensino foi a Prova Brasil aplicada para o ano de 2015, contida nos microdados disponibilizados pelo Sistema de Avaliação da Educação Básica (SAEB) a qual mede o aprendizado escolar, que compreende as provas de português e matemática, aplicadas a alunos de 5 ano, disponibilizado pelo Instituto Nacional de Estudos e Pesquisas Educacionais Anísio Teixeira (Inep), utilizando-se as notas médias obtidas por cada município nos exames, ponderada pelo número de alunos matriculados.

Os dados das variáveis Ineducmae0 e Ineducmae1 referente à escolaridade das mães também foram colhidas no banco de microdados do SAEB. Para efeito de desdobramento da análise, foram aplicados filtros para a variável dependente profmed, para ambas as disciplinas. Previamente na base de dados foram mantidos apenas alunos que participaram da Prova Brasil, já que o banco de dados também contempla a Avaliação Nacional da Educação Básica (ANEB).

Outro filtro aplicado à variável dependente é alusivo ao preenchimento da prova, mantendo-se apenas àquelas observações relativas aos alunos que marcaram sim na opção de preenchimento de prova. Por fim, ainda em relação à variável dependente profmed foram consideradas, para cálculo da proficiência dos alunos, apenas provas que tivessem no mínimo três questões com resposta assinaladas.

Em relação à variável Ingastoeducaluno2, a despesa pública municipal está disposta nos Dados Contábeis dos Municípios, disponibilizados no Finanças do Brasil (FINBRA), publicados pela Secretaria do Tesouro Nacional (STN). O gasto público educacional foi ponderado pelo número de alunos matriculados, revelando uma informação mais apurada no tocante ao real gasto com cada indivíduo. O número de alunos nas redes municipais de ensino fundamental está disponibilizado no EDUDATABRASIL, vinculado ao Inep. No Instituto Brasileiro de Geografia e Estatística (IBGE), foram importados os dados referentes ao Produto Interno Bruto (PIB) municipal para a variável Inpibpercapta.

Os dados das variáveis Inacadpro e Inacadpr1 referente à formação acadêmica dos professores, assim como instescolar referente a estrutura física escolar foram 
colhidos no banco de microdados do Censo Escolar 2015 disponibilizado pelo Ministério da Educação (MEC), por meio da pesquisa declaratória realizada.

Em relação a variável instescolar foram aplicados filtros no tocante a situação de funcionamento, mantendo-se aquelas que apresentaram suas funções em atividade e pleno funcionamento. Ainda sobre instescolar outro filtro aplicado foi alusivo à dependência administrativa, mantendo-se apenas àquelas escolas dependentes da rede municipal de ensino. A amostra final perfez um total de 1530 municípios da região Nordeste.

\section{RESULTADOS}

Para fins de realização das regressões, como variável dependente foi utilizada a proficiência média por município em língua portuguesa para alunos do $5^{\circ}$ ano, como também em matemática, respectivamente para o período de 2015. Os resultados obtidos partem da amostra de 1530 municípios da região Nordeste. Nas tabelas 3 e 4, estão expostos os resultados da estimação do modelo de regressão para os quantis q10, q25, q50, q75 3 q90.

De acordo com a tabela 3, observa-se que a variável Ingastoeduc2, a qual representa o logaritmo natural do gasto público em educação por aluno matriculado na rede municipal de cada município ao quadrado, e objeto principal de explicação do estudo realizado, foi estatisticamente significativa para os quantis q25 e q90 a um nível de significância de $1 \%$.

Tais coeficientes apresentaram um efeito negativo, neste caso levando a especular uma possível não dependência de desempenho escolar, principalmente da despesa com o aluno, abrindo assim uma gama de outros possíveis motivos relacionados ao desempenho escolar, como fatores de gestão de recursos ou fatores familiares. Contudo, como admitiu-se apenas os gastos no período contemporâneo, é possível que seus efeitos não ocorram de forma imediata, mas sim de forma defasada, o que justificaria a ausência do gasto na maioria dos quantis analisados.

A variável Inpibpercapta, que indica o logaritmo natural do PIB per capita de cada município, foi estatisticamente significativa apenas para os quantis mais baixos q10, 
q25 a um nível de significância de 1\%. O resultado positivo obtido dos coeficientes revela o mesmo que o resultado esperado, ou seja, que um maior nível de renda condiga com um melhor desempenho educacional.

Tabela 3 - Proficiência Média por Município em Língua Portuguesa Variáveis Variável Dependente: Profmedlp

\begin{tabular}{|c|c|c|c|c|c|}
\hline & \multicolumn{5}{|c|}{ Quantil $(\boldsymbol{\tau})$} \\
\hline & q10 & q25 & q50 & q75 & q90 \\
\hline Ingastoeduc2 & -.00925482 & $-.010194^{\star * *}$ & -.00139206 & -.02099338 & $-.053493^{* * *}$ \\
\hline Inpibpercapta & $.0710839^{\star * \star}$ & $.0395697^{\star * *}$ & .00382418 & -.00577805 & -.05323804 \\
\hline Ineducmae0 & -.01097046 & -.0345722 & $-.069084^{* * *}$ & $-.0946583^{* * *}$ & $-.1191606^{\star \star \star}$ \\
\hline Ineducmae1 & .02141492 & $.03099823^{\star *}$ & $.0557965^{\star \star *}$ & .00592822 & .01215239 \\
\hline Inacademic0 & -.02420395 & $-.0418713^{\star * *}$ & $-.0535182^{\star * *}$ & -.03654604 & -.01405852 \\
\hline Inacademic1 & $.08084598^{\star \star \star}$ & $.09596063^{* * *}$ & $12925624^{* * *}$ & $15295012^{* * *}$ & $14386374^{\star \star *}$ \\
\hline infescolar1 & .01060029 & $.0045298^{* * *}$ & -.00055865 & -.00355917 & .00232548 \\
\hline infescolar2 & .00654451 & .00400064 & .01041726 & $.02448997^{\star}$ & $.04851626^{* *}$ \\
\hline infescolar3 & -.01537942 & -.01189623 & -.00121024 & -.00506707 & -.00383486 \\
\hline infescolar4 & -.00165838 & .00354708 & .00039473 & -.00696706 & -.00543206 \\
\hline infescolar5 & -.01101706 & -.00190421 & -.00959203 & -.00131446 & -.00297218 \\
\hline infescolar6 & -.00235105 & $-.0049533^{* * *}$ & $-.0068977^{\star \star *}$ & $-.00610079 * *$ & $-.00998737^{\star *}$ \\
\hline infescolar7 & .00016348 & -.00015001 & -.00094055 & $-.0030436^{* * *}$ & $-.0043732^{* \star \star}$ \\
\hline infescolar8 & $-.00432515^{\star *}$ & $.00522361^{* *}$ & $-.0098586^{\star \star \star}$ & $-.0083920^{\star \star \star}$ & $-.008422^{* \star *}$ \\
\hline cons & $-2.368929^{\star \star \star}$ & $-1.853392^{\star * *}$ & $-1.553282^{\star \star *}$ & $-.92302559^{* *}$ & .30721931 \\
\hline
\end{tabular}

Fonte: Elaboração do autor. Legenda: ${ }^{*}$ estatisticamente significativo a $10 \%$; ${ }^{* \star}$ estatisticamente significativa a $5 \%$; ${ }^{* * \star}$ estatisticamente significativa a $1 \%$.

A variável Ineducmae0, indicada pela proporção de mães sem ensino superior por município, foi estatisticamente significativa para os quantis q50, q75 e q90, a um nível de significância de $1 \%$. Tais resultados apresentam um efeito negativo sobre 0 desempenho educacional. Já a variável Ineducmae1, designada pela proporção de mães com ensino superior por município, foi estatisticamente significativa para os quantis q25 e q50 a um nível de significância de 5\% e 1\% respectivamente. Neste caso o efeito apresentado foi positivo. Tais resultados condizem com o efeito geracional advindo da educação, que sinalizam que pais educados tendem a ter filhos com maior nível de educação. $O$ fato que mães serem mais escolarizadas contribui mais com o desempenho dos filhos do que mães menos escolarizadas.

A variável Inacadêmic0 a qual representa a proporção de professores sem ensino superior por município, assim como a variável Ineducmae1, também se mostrou 
estatisticamente significativa para os quantis q25 e q50, a um nível de significância de $1 \%$ para os dois quantis, apresentando um efeito negativo para ambos os coeficientes.

Já a variável Inacadêmic1, que representa a proporção de professores com ensino superior por município, mostrou-se estatisticamente significativa para todos os quantis, apontando um efeito positivo sobre o desempenho escolar.

A interpretação desses resultados para formação acadêmica dos professores se assemelha à interpretação anterior para as variáveis de formação acadêmica das mães. É razoável admitir que professores com ensino superior, estão mais qualificados academicamente para instruir e lecionar do que professores sem ensino superior.

As variáveis infescolar estão relacionadas às instalações físicas das escolas. O objetivo dessa variável foi de dispor a frequência de escolas que apresentavam o mínimo de instalações relevantes para o desenvolvimento das atividades de ensino e aprendizagem por município. As instalações físicas em questão foram banheiro, cozinha, sala da diretoria, sala do professor, biblioteca, quadra de esportes, laboratório de informática e laboratório de ciências.

Foi construída uma escala da variável infescolar, a qual se inicia na infescolar1 até a infescolar8. Respectivamente a primeira variável infescolar1 é composta pela frequência das escolas que contém todas as instalações físicas citadas relevantes para o estudo por município. As variáveis subsequentes contêm a frequência das escolas com uma instalação física a menos, ordenadas por grau de importância, que corresponde ao inverso da ordem citada anteriormente na descrição das instalações físicas, até que a última variável infescolar8 contenha apenas uma única instalação física.

A variável infescolar1 foi estatisticamente significativa apenas no quantil q25 a um nível de significância de 1\%, apresentando um efeito positivo para o desempenho educacional. Assim como a variável infescolar2 que também apresentou um efeito positivo para o desempenho escolar e foi estatisticamente significativa nos quatis mais altos q75 e q90, porém apenas a um nível de significância de 10\% e 5\%, respectivamente. 
A variável infescolar3, assim como infescolar4 e infescolar5 não foram estatisticamente significativas em nenhum dos quantis utilizados. A variável infescolar6 foi estatisticamente significativa a $1 \%$ para os quantis q25 e q5a; e à $5 \%$ nos quatis q75 e q90. A variável infescolar7 assim como a variável infescolar2 foi estatisticamente significativa apenas nos quatis mais altos q75 e q90, porém a um nível de significância de a $1 \%$. A variável infescolar8 foi estatisticamente significativa a $1 \%$ nos quantis q50, q75 e q90; e à $5 \%$ nos quatis q10 e q25.

Portanto, levando em conta que quanto mais instalações físicas de maior necessidade houver em cada escola por município, a relação com o desempenho escolar é positiva, ou seja, pode haver um maior aprendizado dos alunos em cada escola. Na medida em que diminui o número de instalações as quais vão mantendo-se apenas aquelas cada vez mais primordiais para as atividades de ensino e estudo, a relação com o desempenho se inverte possivelmente reduzindo o nível de aprendizado dos alunos.

Discutidos os resultados do desempenho educacional em língua portuguesa, passa-se a resultado do desempenho em matemática. De acordo com a tabela 4 , observa-se que a variável Ingastoeduc2, foi estatisticamente significativa para o quantil q90 a um nível de significância de 5\%, apresentando um efeito negativo em relação ao desempenho escolar.

O fato de que, para esta variável, poucos quantis sejam estatisticamente significativos, o que também se repete na estimação para a proficiência em língua portuguesa, reafirma a especulação de que possivelmente o gasto público em educação realizado em 2015 não explique o desempenho escolar dos alunos para este ano, podendo o mesmo refletir no desempenho nos anos seguintes, ou seja, de forma defasada.

Ainda, é possível conjecturar que talvez fatores de gestão estejam associados a tal problemática. Uma possível explicação que pode ser inferida é que os gastos realizados provavelmente não estão sendo utilizados eficientemente, fazendo com que uma elevação do mesmo piore a situação em que se encontra a educação, levando a 
um maior uso ineficiente dos recursos disponíveis, explicando assim o efeito negativo apresentado pelos coeficientes das regressões.

Tabela 4 - Proficiência Média por Município em Matemática

\begin{tabular}{|c|c|c|c|c|c|}
\hline \multirow{3}{*}{ Variáveis } & \multicolumn{5}{|c|}{ Variável Dependente: Profmedmt } \\
\hline & \multicolumn{5}{|c|}{ Quantil ( $\boldsymbol{\tau})$} \\
\hline & q10 & q25 & q50 & q75 & q90 \\
\hline Ingastoeduc2 & -.00628163 & -.00359386 & .0091582 & -.00253891 & $-.05008161^{\star *}$ \\
\hline Inpibpercapta & $.03913107^{\star *}$ & $.02452398^{*}$ & -.01318984 & -.03586664 & $-.104868^{* * *}$ \\
\hline Ineducmae0 & -.01768193 & -. $0162181^{*}$ & -.03109808 & -.04207299 & -.08230472 \\
\hline Ineducmae1 & .01004719 & $.01687326^{*}$ & .02398128 & .02571116 & $-.0006128^{* *}$ \\
\hline Inacademic0 & $-.01974357^{\star}$ & $-.047352^{* \star *}$ & $-.0620396^{\star * *}$ & $-.04866425^{\star *}$ & -.01218357 \\
\hline Inacademic1 & $.0749167^{* * *}$ & $.0880797^{\star \star *}$ & $.119035^{\star \star \star}$ & $.1218936^{\star \star *}$ & $.1336418^{\star * *}$ \\
\hline infescolar1 & .00689214 & .00668362 & .00020867 & -.01038182 & .0480519 \\
\hline infescolar2 & .0048941 & .00313325 & .00824984 & .02716627 & $.04096873^{*}$ \\
\hline infescolar3 & -.01110859 & - .00701258 & .00110866 & .00577883 & -.00461058 \\
\hline infescolar4 & -.00379952 & - .00188628 & .00156347 & -.00385237 & -.01111146 \\
\hline infescolar5 & -.00351497 & - .00395533 & -.00757666 & -.00579114 & -.00296303 \\
\hline infescolar6 & $-.0040602^{* *}$ & $-.004881^{* \star *}$ & $-.0074960^{\star \star *}$ & $-.0089948^{* \star *}$ & $-.0115216^{* * *}$ \\
\hline infescolar7 & -.00019514 & .00022456 & -.00074807 & $-.0033167^{\star * *}$ & $-.0046866^{* \star *}$ \\
\hline infescolar8 & $-.0047888^{\star \star *}$ & $-.004570^{\star * \star}$ & $-.0067968^{* * *}$ & $-.00699054^{\star *}$ & $-.0081886^{\star \star *}$ \\
\hline cons & $-1.76095^{\star \star \star}$ & $-1.53664^{* * *}$ & $-1.317227^{\star * *}$ & $-.76672794^{\star *}$ & .88121121 \\
\hline
\end{tabular}

Fonte: Elaboração do autor. Legenda: * estatisticamente significativo a $10 \%$; ${ }^{* *}$ estatisticamente significativa a $5 \%$; ${ }^{* * *}$ estatisticamente significativa a $1 \%$.

O fato de que, para esta variável, poucos quantis sejam estatisticamente significativos, o que também se repete na estimação para a proficiência em língua portuguesa, reafirma a especulação de que possivelmente o gasto público em educação realizado em 2015 não explique o desempenho escolar dos alunos para este ano, podendo o mesmo refletir no desempenho nos anos seguintes, ou seja, de forma defasada.

Ainda, é possível conjecturar que talvez fatores de gestão estejam associados a tal problemática. Uma possível explicação que pode ser inferida é que os gastos realizados provavelmente não estão sendo utilizados eficientemente, fazendo com que 
uma elevação do mesmo piore a situação em que se encontra a educação, levando a um maior uso ineficiente dos recursos disponíveis, explicando assim o efeito negativo apresentado pelos coeficientes das regressões.

A variável Inpibpercapta foi estatisticamente significativa a um nível de significância de $1 \%$ para o quantil q90, a $5 \%$ para o quantil q10 e a $10 \%$ para o quantil q25. Como também obtido para a regressão em língua portuguesa, os resultados positivos dos coeficientes revelam que municípios com um maior PIB possivelmente elevem o desempenho dos alunos.

O fator social possivelmente é o explicador de tal fato, a região Nordeste a qual este estudo é feito, dentre as regiões do Brasil, se encontra em meio aos piores resultados econômicos do país, com baixos nível de renda e submetido a condição de vida precária. Diferente de regiões ou localidades com melhores níveis econômicos e sociais, o Nordeste, por ser característica sua precária situação econômica, reage a uma melhora de seus rendimentos diferente de localidades com situação econômica superior. Possivelmente, tal alteração em regiões mais abastadas, não tenha a mesma relevância que em regiões menos abastadas, explicando possivelmente assim o efeito positivo do resultado encontrado para a variável.

A variável Ineducmae0 foi estatisticamente significativa apenas a um nível de significância de $10 \%$ para o quantil q25. Tal resultado apresentar um efeito negativo sobre o desempenho educacional conforme esperado e ocorrido também na regressão para língua portuguesa. Já a variável Ineducmae1 foi estatisticamente significativa para os quantis q25 e q90 a um nível de significância de $1 \%$ e 5\%, respectivamente. Neste caso, o efeito apresentado foi positivo conforme esperado e interpretado também na regressão para língua portuguesa. Assim, ter mães com maior nível de escolaridade ajuda a elevar o desempenho escolar de seus filhos, talvez pelo fato de ter um maior conhecimento da situação em que seu filho esteja inserido, oferecendo melhor orientação como também apoio emocional.

A variável Inacadêmico se mostrou estatisticamente significativa para os quantis q25 e q50, a um nível de significância de 1\%, para q75 a 5\% e para q10 a 10\%. Para todos os quantis citados, o efeito apresentando para com a variável dependente é 
negativo. Já a variável Inacadêmic1, assim como na regressão para língua portuguesa, mostrou-se estatisticamente significativa para todos os quantis reafirmando fortemente um efeito positivo da formação superior dos professores sobre o desempenho escolar dos alunos. Tal variável apresentou os resultados mais sólidos e com maior poder de explicação para o estudo em questão. A interpretação desses resultados para formação acadêmica dos professores com nível superior implica em um possível maior desempenho escolar dos alunos.

A variável infescolar1, assim como infescolar3, infescolar4 e infescolar5 não foram estatisticamente significativas em nenhum dos quantis utilizados. A variável infescolar2 foi estatisticamente significativa apenas a $10 \%$ para o quantil q90.

A variável infescolar6 foi estatisticamente significativa a $1 \%$ em todos os quatis exceto no quantil mais baixo q10, este foi estatisticamente significativo a um nível de significância de $5 \%$, apresentando conforme na regressão para língua portuguesa, um efeito negativo.

A variável infescolar7 foi estatisticamente significativa para os quantis mais altos q75 e q90 a um nível de significância de 1\%. E a variável infescolar8 foi estatisticamente significativa em todos os quantis a $1 \%$, ambas apresentando um efeito negativo para com o desempenho educacional.

A interpretação dos resultados das variáveis de infraestrutura escolar se assemelha em ambos os casos das regressões realizadas. Percebeu-se o diferencial que para a regressão em matemática o efeito positivo apresentado para as varáveis com maior número de instalações como infescola1 e infescolar2 não foi estatisticamente significativo, porém foi reafirmado o efeito negativo causado pelas variáveis com um menor número de instalações como infescolar6 e infescolar8, as quais vão mantendo-se apenas aquelas instalações cada vez mais primordiais para as atividades de ensino e estudo, possivelmente reduzindo o nível de aprendizado dos alunos. 


\section{CONSIDERAÇÕES FINAIS}

Dadas as discussões realizadas ao longo deste trabalho, com base nos resultados obtidos, não foi possível identificar, o efeito contemporâneo do gasto público em educação por município, para o ano de 2015, sobre o desempenho escolar médio de alunos nordestinos em testes padronizados de português e matemática.

Tal fato explica-se pela pequena quantidade de quantis estatisticamente significativos e pode estar relacionado a um efeito defasado da variável gasto para com a variável dependente de desempenho escolar dos alunos. É razoável que o efeito de um aumento dos gastos em educação repercuta em momentos futuros, principalmente, aqueles ligados à infraestrutura, capacitação e gestão administrativa, dado que os mesmos requerem um período de maturação.

O PIB per capita mostrou-se relevante, principalmente, para os quantis mais baixos da distribuição, evidenciando que o efeito renda é mais favorável naqueles municípios cujo desempenho escolar se mostra mais baixo.

Ademais se observou uma forte relação a qual diz respeito ao poder de explicação do desempenho educacional por meio da formação acadêmica dos professores. Tal relação implica que professores com formação acadêmica com nível superior apresentem um resultado mais significativo do seu trabalho, em relação à aqueles com formação acadêmica inferior.

Pode-se perceber, em relação à variável escolaridade das mães, que o efeito apresentado pela variável nas regressões foi condizente para com o esperado. $O$ resultado observado indica que níveis de escolaridades das mães mais elevados, possivelmente eleva o desempenho educacional dos filhos. Tal fato pode estar ao efeito Inter geracional da educação, como também pode relacionar-se à importância do apoio dos pais em relação a compreensão das dificuldades e necessidades dos filhos no processo de aprendizagem. 
Finalmente, destaca-se o efeito da infraestrutura da escola sobre o desempenho escolar, dando destaque para aquelas mais bem aparelhadas. Portanto, de acordo com o cenário tratado, um melhor nível de estrutura condiz com um melhor desempenho.

\section{REFERÊNCIAS}

ALMEIDA, Ivan Castro. Gastos com educação no período de 1994 a 1999. Revista brasileira de estudos pedagógicos, v. 82, n. 200-01-02, 2007.

BARROS, Ricardo Paes de et al. Determinantes do desempenho educacional no Brasil. 2001. Disponível em: <http://repositorio.ipea.gov.br/handle/11058/5065>. Acesso em 29 set. 2017.

- $O$ impacto de gestão escolar sobre o desempenho educacional. Washington: BID, 1997b, 39p (Série Documentos de Trabajo de la Rede de Centros/BID, 301).

BRASIL. Constituição (1988). Constituição da República Federativa do Brasil, promulgada em 5 de outubro de 1988. São Paulo: IMESP, 2016.

CASTRO, Jorge Abrahão de; DUARTE, Bruno de Carvalho. Descentralização da educação pública no Brasil: trajetória dos gastos e das matrículas. 2008. Disponível em: < http://repositorio.ipea.gov.br/handle/11058/1530>. Acesso em: 22 set. 2017.

FELÍCIO, Fabiana; FERNANDES, Reynaldo. 0 efeito da qualidade da escola sobre o desempenho escolar: uma avaliação do ensino fundamental no estado de São Paulo. IN: Anais do XXXIII Encontro Nacional de Economia, 2005.

FONSECA, Marília. Políticas públicas para a qualidade da educação brasileira: entre 0 utilitarismo econômico e a responsabilidade social. Cadernos Cedes, Campinas, v. 29, n. 78, p. 153-177, 2009.

HANUSHEK, Eric A. Alternative School Policies and the Benefits of General Cognitive Skills. Economics of Education Review, v. 25, n. 4, Aug. 2006, p. 447-462. 2017.

HEDGES, L.; LAINE, R. D.; GREENWALD, R. Does money matter?: A meta-analysis of the effects of differential schools inputs on student outcomes. Educational Researcher, v. 23, n. 3, p. 5-14, Apr. 1994.

. The effect of school resources on student achievement. Review of Educational Research, Washington, DC, v. 66, p. 361-396, 1996a.

. Interpreting research on school resources and student achievement: a rejoinder to Hanushek. Review of Educational Research, Washington, DC, v. 66, p. 411-416, 1996b. 
KOENKER, Roger; BASSETT JR, Gilbert. Regression quantiles. Econometrica: journal of the Econometric Society, p. 33-50, 1978.

POCHMANN, Marcio. Educação e trabalho: como desenvolver uma relação virtuosa. Educação e Sociedade, v. 87, n. 25, 2004.

SEGNINI, Liliana Rolfsen Petrilli. Educação e trabalho: uma relação tão necessária quanto insuficiente. São Paulo em Perspectiva, v. 14, n. 2, p. 72-81, 2000.

SOARES, Robson Fernandes; CLEMENTE, Ademir. Relação entre gastos com educação e desempenho escolar: um estudo nos municípios paranaenses no período de 2005 a 2011. IN: Anais do Congresso Brasileiro de Custos-ABC. 2013.

SOARES, Sergei; SÁTYRO, Natália. O impacto de infra-estrutura escolar na taxa de distorção idade-série das escolas brasileiras de ensino fundamental: 1998 a 2005. Texto para Discussão, Instituto de Pesquisa Econômica Aplicada (IPEA), 2008.

Recebido em: Junho de 2018

Aceito em: agosto de 2018 